Third International Engineering Systems Symposium

CESUN 2012, Delft University of Technology, 18-20 June 2012

\title{
Game-like Characteristic of Engineering Design
}

\author{
Sertac Oruc and Scott W. Cunningham \\ Policy Analysis Section, Faculty of Technology, Policy and Management, Delft University \\ of Technology, Jaffalaan 5, 2600 GA, Delft, The Netherlands \\ S.Oruc@tudelft.nl, S.Cunningham@tudelft.nl
}

\begin{abstract}
Engineering design is conventionally regarded as a mono actor optimization problem and modeled accordingly. Decision making, values and optimality are building blocks of conventional engineering design. However with the advent of decentralized decision making processes, various actors are more likely to be involved in decision making processes in engineering design. As a response in this paper we attempt to claim that engineering design is inherently multi actor and has game-like characteristics. Accordingly a research agenda is put forward.
\end{abstract}

Keywords. Multi Actor Systems, Engineering Design, Game Theory

\section{Introduction}

In the process of designing engineering components and systems, optimization approach has been the conventional choice. In this context we regard optimization as formulation and execution of problems instead of the narrower use of the word as a mathematical technique. As a mathematical technique, optimization is a pragmatic tool and an important step to bring about the final design, although not in focus of our discussion.

In our context engineering design refers to the plan for construction of an engineering artifact. It is a specification of the artifact intended to accomplish a certain designated goal. And optimization is the traditionally applied approach to guide this process. Engineering design, being based on optimization, is directly related to value paradigm and decision theory.

Decision making, and therefore decision theory, is directly employed in the current theory and practice of engineering design. It is important to distinguish theory of engineering design and theory of engineering modeling at this point as the latter aims to asses and predict the performance of engineering artifact whereas the former is related to the total design of the artifact anew.

Multi actor engineering design refers to the engineering design that involves more than one actor. In our framework we consider initially a customer who funds and 
utilizes the design artifact and a designer who actually designs the engineering artifact. However the conceptual framework we consider here can be applied other type of business models that involve various other type of actors.

In the following section we give the engineering design in terms of optimization problem. A methodological review is presented in this section. Later game like character of engineering design is presented and it is formalized with a game theoretic model. In the subsequent section new approaches to engineering design is discussed briefly. Lastly concluding remarks and reflections are presented

\section{Engineering Design Optimization Problem}

Engineering Design is historically seen as a mono actor optimization problem. Here in this section we present the conventional approach to engineering design and discuss a brief methodological review on this issue. Revisiting conventional approach makes it possible to extend the framework to equilibrium approach in the subsequent sections.

Conventionally engineering design is associated with giving the "appropriate" design decisions. Appropriateness in this context is determined by an optimization process, which is sometimes only intuitive and sometimes grounded on rational choices based on values and analytical techniques (Siddall, 1982). The decision making in engineering design process begins with the very basic components such as the kind of steel to be used or engine oil to be utilized. These basic component decisions permit certain measures for design characteristics such as engine power, machine weights etc. Then the design characteristics constitute various values that are considered to be maximized or minimized according to the "wishes" of the designer. Optimization of the engineering design may hold for the case where only one designer and accordingly one wish is at stake. How to handle the situation in case there are more than one designer or choice is the central question of this paper.

\subsection{Optimization Concepts}

Consider the following linear program, where $\mathrm{x}$ are decision variables, $\mathrm{c}$ parameterizes the objective function, and $\mathrm{A}$ and $\mathrm{b}$ parameterize the constraints.

$$
\begin{aligned}
\min _{x} Z=c^{T} x & \\
\text { subject to } A x & \geq b \\
x & \geq 0
\end{aligned}
$$

The solution to this optimization problem can be expressed in terms of a set of algebraic equations; indeed, this is the core of the simplex algorithm. Investigation of the solutions to this set of variables reveals a fundamental insight that only the set of linear operations (addition and subtraction) are needed to find the optimum solution. Furthermore, the derivation of any solution can be exclusively expressed in terms of its slack variables. This fundamental insight leads immediately to the duality theory of linear programming. Furthermore, the use of the dual program is useful since it 
provides insight into the trade-offs at the optimum, without requiring extensive reoptimization of the problem from scratch (Hillier et al., 1990).

The vector of decision variables $\mathrm{x}$ is an optimum solution to the problem if the vector is a feasible solution, and if there is a dual vector $y$. The vector $y$ must satisfy the following optimization problem:

$$
\begin{array}{r}
\max _{y} Z=b^{T} y \\
\text { subject to } A^{T} y \leq c \\
y \geq 0
\end{array}
$$

This linear optimization problem can be transformed, with no loss of generality, in various ways. The maximization can be reduced to a minimization, the constraint equalities can be transformed into inequalities, and the constraint boundaries transformed from in various forms. There is an account of this point in (Hillier and Lieberman, 1990), but also in (Papalambros and Wilde, 2000).

\subsection{Engineering Design Optimization}

Consider the following model of the design process. A designer has $i=\{1, \ldots, m\}$ design options. A customer has $\mathrm{j}=\{1, \ldots, \mathrm{n}\}$ criteria for choice. The customer expresses a weighting of their preferred criteria, $\mathrm{w}_{1} \ldots \mathrm{w}_{\mathrm{n}}$. The designer has a score card, a matrix $U$ which is dimensioned $i \times j$. Each element of the score card, $\mathrm{u}_{\mathrm{ij}}$, expresses the utility obtained by the designer for selecting a particular technological option. Utilities are a function of expressed customer satisfaction $\left(\mathrm{w}_{\mathrm{j}}\right)$ minus the implementation cost $\left(\mathrm{k}_{\mathrm{ij}}\right)$. Thus $\mathrm{u}_{\mathrm{ij}}$ can be expressed using the following function

$$
u_{i j}=\left(w_{j}-k_{i j}\right)
$$

The designer's choice is to combine or blend technological functions so as to achieve maximum utility. This involves playing a mixed strategy, $\mathrm{v}_{1} \ldots \mathrm{v}_{\mathrm{n}}$. The design problem is conditioned on the designer's knowledge of the game matrix $\mathrm{U}$, the costs of implementation $\mathrm{k}_{\mathrm{ij}}$, and the preferences of the customer $\mathrm{w}_{\mathrm{j}}$.

This problem, given fixed preferences of the customer, reduces to the following classic expression of an engineering design problem.

$$
\begin{gathered}
\max _{v} \mathrm{Z}=\sum_{i=1}^{m} \sum_{j=1}^{n} v_{i} u_{i j} \\
\text { subject to } \sum_{i=1}^{m} v_{i}=1, \\
v_{i} \geq 0 .
\end{gathered}
$$


This optimization model implies a mono actor, monoobjective problem. This actor objective problem constitutes one side of the multi actor problem. Unilaterally formulated optimization problems from various actors constitute multi actor problem. Later we show how the above listed mono actor optimization model is fit into multi actor problem.

\subsection{Methodological Review}

The use of formal optimization modeling in engineering literature dates at least back to the early 1970s (Lifson, 1972; Siddall, 1982, p. 13). Most expressions of the problem involve non-linear optimization, although the use of linearization procedures as a means of approximation are common (Papalambros and Wilde, 2000, p. 131). Variant expressions of the problem include multi-objective optimization, as well as optimization under risk (Buede, 2000, ff. 164). Others (Cummings, 2006) question the engineering optimization paradigm on fundamental questions of value.

Infrastructure problems of design, planning, allocation and control all invite the use of optimization techniques (Flintsch and Chen, 2004). Consider for instance the canonical example of optimization, linear programming. Despite the considerable usefulness of linear programming in decision-making, this technique depicts the system preferences of just a single actor. Arguably, the most apt use of linear programming has been in command and control environments such as planned economies, the military, and oligopolistic corporations. In these environments there is less capacity for the system to rebound in response to decisions or tactics. Linear programming is one of the premier tools for computer modelling of decision-making under constraint. Linear programming, and its extensions, is widely applied across many fields of application. The principle computer algorithm for the technique was discovered by Dantzig in 1948, as reported in a memorandum of the United States Air Force (Dantzig, 1998). Linear sensitivity analysis, and ultimately a full expression of a strategic theory of games, emerged organically from this initial research in linear programming (Cunningham, 2008).

Variant Statements of the Problem. Research into engineering optimization has been productive, resulting in a burgeoning literature. Previous research, less than ten years old, provides a survey of multi-objective optimization in engineering (Marler and Arora, 2004).

This survey excludes many of the technical details and, instead, provides a road map of currently available continuous nonlinear methods and literature. General concepts are briefly described, and references are included for further investigation. In addition, this paper consolidates seemingly different concepts, methods, and terminology stemming from diverse applications (p. 369).

The fact that this work has been cited by more than 189 authors (Web of Science, 2012) suggests that the question of multi-objective optimization remains of essential interest to designers, and is still subject to rapid progress. Further, the field requires 
multi-disciplinary surveys to keep abreast of the varied technical details from the various literatures.

Modern infrastructure supports the exchange of multiple kinds of goods and services. Flows are becoming multi-commodity, multi-modal, multi-sectoral and multi-faceted. For instance, the shipping industry has long been a multi-commodity enterprise. Multi-modal traffic is increasingly being considered, for instance, in the design of rail links. Multi-faceted infrastructure is leading to an explosion of new consumer options, and new concerns for decision-makers. For instance, consumers may choose "green" electricity: this formerly homogeneous good grows more diversified as consumers are presented with increasing amounts of information about the environmental impacts of their choices. Thus, network infrastructures are inherently multi-objective in character.

Multi-Actor Design Problems. Despite this interest in formal models of multiobjective optimization, very little synthesis and review is available on the related but distinct question of multi-actor design. As evidenced by the multi-objective setting, there is a proliferation of specialized applications, rich in technical detail, in the multiactor design setting. There however has been little effort in consolidation. Two exceptions are (Cunningham, 2008), and (Cunningham and van der Lei, 2009).

Infrastructure is inherently multi-actor in character. Infrastructure is commissioned, designed and built, and utilized by multiple stakeholders. There is no single objective function held in common between these stakeholders; rather, an expression of opposing economic, social or physical forces is a more useful paradigm for expressing network usage. This realization is often a better depiction of system behavior. In network infrastructures - such as highways, airports, water systems, electrical transmission and distribution systems - there are always multiple actors making multiple if interdependent decisions.

Our question concerns whether the classic design problem continues to hold in a multi-actor setting. Can design effectively be expressed as an optimization problem, even in the simplest of multi-actor settings? Can the design problem be reformulated to better encompass a fully multi-actor setting? If so, what are the consequences for engineering practice?

\section{The Game-Like Character of Engineering Design}

In this paper the game like character of multi actor engineering design is emphasized. Theory of games traditionally analyzes multi actor behavior in situations where individual interests conflict or coincide with each other. Actors in scrutiny are considered to be interested in their own pay off function and alter their strategies in order to maximize their utilities. Von Neuman and Morgenstern's seminal work Theory of Games and Economic Behavior (Von Neumann and Morgenstern, 1944) laid down the mathematical foundations of game theory, which triggered various theories around the idea of equilibrium instead of optimum. 
In this section we hypothesize that engineering design could be considered as an equilibrium problem instead of an optimization problem unlike the conventional approach to the field.

\subsection{Previous Work}

Social choice theory stands as a prominent field in this regard. Collective decision making influence and determine various outcomes of formal institutions, i.e. laws. "voting paradox" and Arrow's "impossibility theorem" are two generally acknowledged.

Arrow's impossibility theorem implies that when voters have more than two distinct options no voting system can convert ranked individual preferences into a collective ranking while also meeting a specific set of criteria (Arrow, 1950). These criteria can be listed as;

- Non-dictatorship: No single member's choices should prevail in every voting instance. The social welfare function should account for multiple voters' wishes.

- Universality: The function should yiled a unique and complete ranking of societal choices.

- Independence of irrelevant alternatives: Changes in individuals' rankings of irrelevant alternatives of a certain subset should have no impact on the collective ranking of the subset.

- Pareto efficiency (Unanimity): If every voter prefers a certain option to another then the collective preference must make the same preference.

Arrow's impossibility theorem points out that if the decision making group of individuals. Various formal and informal proofs follow in literature (Hansen, 2002; Geanakoplos, 1996).

Arrow's impossibility theorem showcase one particular case where social processes build up game like consequences. The implication of impossibility theorem in engineering design is that the different interests of different actors in a multi actor engineering design setting satisfaction of all individual choices of the actors is less likely than intuition may suggest. A thorough examination in this regard is usually required.

Following the formal modeling approach in game theoretical analysis in the next sub section we give an effort to present engineering design problem in formal model format. Creating a formal model of the problem would enable policy testing and operational insight into engineering design. 


\subsection{A Formal Model of Engineering Design}

The first step in formulating the game-like setting of design activities is to first specify the rules of the game. These rules may be presented by means of the "order of play" (Rasmussen, 2007, p. 14). The essential elements of a game, that is players, actions, payoffs and information should be attributed in the model.

\section{A Game of Multi-Actor Design}

\section{Rules}

1. The designer presents the customer a set $\mathrm{i}=\{1, \ldots, \mathrm{m}\}$ of technological options. Included in this option are one or more status-quo options (resulting in the use of alternative technologies), and a null option (resulting in a failure to adopt any technology at all). The customer knows their own utilities (X), as well as the costs of the designer.

2. The designer elicits preferences from the customer, requesting a set of weights or utility scores for each design criteria, $w_{j}$.

3. The designer completes the design, and the artifact is developed or implemented, thereby becoming available to the customer.

4. The customer then expresses their true preference for the product by choosing between the finished product and the null option.

\section{Pay-Offs}

The customer receives utility $\mathrm{x}_{\mathrm{ij}}$ for objective $\mathrm{j}$, under option $\mathrm{i}$. The choice of the null option is set to zero for all criteria, with no loss of generality. The designer's pay-offs are as stated previously.

We offer two propositions concerning engineering design and an extended game between designers and customers. The first concerns the efficacy of engineering optimization in a multi-actor setting. The second concerns the capability of obtaining truthfully stated preferences from customers prior to the design process.

Proposition 1. The multi-actor design game rarely results in optimization problem.

Proof. The minimax theorem, which is closely related to the strong duality principal of linear programming, demonstrates that any zero-sum game is reducible to an optimization problem expressed in minimax form. The proposition restated is therefore the question of whether the multi-actor design game is inherently zero sum. This is tantamount to asking whether there must always be a proportional relationship between customer utility and the cost to the designer. 
Corollary. In those cases where optimization is an effective expression of the design problem, this will be because there is a direct transfer of utility from the customer to the designer in proportion to technological performance. This can occur only in those cases where it is possible to fully and completely valorize product performance.

Proposition 2. Stated preferences, given the rules of the game, are rarely those actually expressed by customers.

Definition (Strategic Complements). Strategic complements occur whether one player acts it encourages the other player to do more of the same.

Definition (Last-Mover Advantage). A last-mover advantage occurs whether taking the first action in the game benefits the player. Gal-Or (1985) provides a generic result concerning the relationship between strategic complements and last-mover advantages. There are always last-mover advantages where there are strategic complements.

Proof. Since the utility of the designer is proportional to the interests of the customer, there are strategic complements in this game. Since there are strategic complements, there must also be a last mover advantage for the customer. Because there is a last mover advantage, the initial expression of values by the customer cannot be in equilibrium. This follows directly from the definition of the Nash equilibrium.

Corollary. It follows from the revelation principal that it is not incentive compatible for the customer to fully reveal their preferences in the presence of the designer prior to the actual design of the product.

The model can be expanded in various ways. It could be revised to be a game in continuous strategies. This would be more general, without reducing any of the findings. Also the model can deal with incomplete information situations such as the case when the customer knows about the engineering costs.

\section{New Approaches to Engineering Design}

In engineered systems equilibrium is ever present. Mechanical, thermal, electrical, fluidic - all these systems are engineered for a robustness requirement determined by some specifications. The specifications vary depending on the nature of the environment that the engineered artifact is built or utilized in. For instance, in construction sector requirement of resilience to external disturbances such as earthquakes is different in Japan compared to the Netherlands. However in both cases some specifications are designed and propagated. The equilibrium concept that guarantees a certain specification is always employed in engineered systems. In addition, social equilibrium is an extremely useful concept that has seen application in both economics and the theory of games. Inarguably, network equilibrium is an important and useful concept for analysis and design. We do however draw a cautionary note, in recommendations for future research, about the appropriate use of equilibrium analysis. 


\section{Conclusions}

In this paper a new approach to engineering design is debated. The traditional approach to engineering design is contested with a multi actor approach that emphasize game theoretical modeling. With this approach the top down characteristic of engineering design optimization is challenged with a decentralized approach of equilibrium. Multiple actors which involve customers, funders and suppliers etc as well as designer themselves is considered as opposed to mono actor (i.e. designer) setting.

Engineering design is historically been considered as a mono actor multi criteria decision problem. Decision models, which most of the time emphasize optimization based approaches leave their place to equilibrium models where multiple actors take part in decision making process. Game theoretical concepts have increasing importance in analyzing such decision processes. In this paper we attempt to formulate the game like characteristic of the engineering design problem.

New methods provide better insights as they encompass multi actor processes. A readily available multi actor theories can be applied in engineering design as opposed to traditional optimization modeling.

Finally we see a pathway for continuation of laid down approach to engineering design. This work as presented here is to be expanded with an articulated model in continuous strategies form. The implications of the model for engineering design is also to be articulated and discussed. The model could be articulated within incomplete information settings, which is a widely encountered case in engineering design.

\section{References}

Arrow, K. J. (1950), "A difficulty in the concept of social welfare", The Journal of Political Economy, vol. 58, pp. 328-346.

Buede, D. M. (2000), The Engineering Design of Systems: Models and Methods, John Wiley and Sons: New York.

Cummings, M. L. (2006), "Integrating ethics in design through the value-sensitive design approach," Science and Engineering Ethics, vol. 12, iss. 4, pp. 701-715.

Cunningham, S. W. and T. van der Lei (2006), "Decision-making for new technology: A multi-actor, multi-objective method," Technological Forecasting and Social Change, vol. 76 , iss. 1 , pp. 26-38.

Cunningham, S. W. (2008), "A review of complementarity problems for the design and analysis of infrastructure," Proceedings of the Infrastructure Systems and Services Conference, 10-12 November, Rotterdam: The Netherlands.

Dantzig, G. (1998). Linear programming and extensions. Princeton Univ Press: Princeton. 
Flintsch, G. and Chen, C. (2004). "Soft computing applications in infrastructure management." Journal of Infrastructure Systems, 10, 157.

Gal-Or, E. (1985), "First mover and second mover advantages," International Economic Review, vol. 26, no. 3: 649-653.

Geanakoplos J. (1996) “Three Brief Proofs of Arrow's Impossibility Theorem”, manuscript, Cowles Foundation, Yale University, New Haven, CT, 1996

Hansen P. (2002), “Another Graphical Proof of Arrow's Impossibility Theorem,” Journal of Economic Education.

Hillier, F., Lieberman, G., and Hillier, M. (1990). Introduction to operations research. McGraw-Hill Singapore.

Lifson, M. W. (1972), Decision and Risk Analysis for Practicing Engineers, Barnes and Noble, New York.

Marler, R. T. and J. S. Arora (2004), "Survey of multi-objective optimization methods for engineering." Structural and Multidisciplinary Optimization, vol. 26, pp. 369-395.

Papalambros, P. Y. and D. J. Wilde (2000), Principles of Optimal Design: Modeling and Computation, Second Edition, Cambridge University Press: Cambridge.

Rasmussen, E. (2007), Games and Information: An Introduction to Game Theory, $4^{\text {th }}$ Edition, Blackwell Publishing: Malden, Massachusetts.

Siddall, J. N. (1982). Optimal Engineering Design: Principals and Applications, Marcel Dekker: New York.

Von Neumann, J., and Morgenstern O. (1944), Theory of Games and Economic Behavior, Princeton: Princeton University Press. 